\begin{tabular}{|c|c|c|c|}
\hline \multirow{2}{*}{$\begin{array}{r}\text { Case Reports in } \\
\text { Gastroenterology }\end{array}$} & \multicolumn{2}{|c|}{ Case Rep Gastroenterol 2017;11:576-583 } & \multirow[b]{2}{*}{$\begin{array}{l}\text { Karger } \\
\text { Open access }\end{array}$} \\
\hline & $\begin{array}{l}\text { DOI: } 10.1159 / 000480375 \\
\text { Publisned online: September 27, } 2017\end{array}$ & $\begin{array}{l}\text { The Author(s) } \\
\text { Published by S. Karger AG, Basel } \\
\text { www.karger.com/crg }\end{array}$ & \\
\hline & $\begin{array}{l}\text { This article is licensed under the } \mathrm{Cr} \\
\text { International License (CC BY-NC) (ht } \\
\text { Usage and distribution for commercial }\end{array}$ & $\begin{array}{l}\text { nons Attribution-NonCommercia } \\
\text { ger.com/Services/OpenAccessLice } \\
\text { uires written permission. }\end{array}$ & \\
\hline
\end{tabular}

\title{
Hepatectomy for Hilar Cholangiocarcinoma with Right-Sided Ligamentum Teres Using a Hepatectomy Simulation System
}

\author{
Seikan Hai Etsuro Hatano Tadamichi Hirano Yasukane Asano \\ Kazuhiro Suzumura Hideaki Sueoka Jiro Fujimoto \\ Department of Surgery, Hyogo College of Medicine, Nishinomiya, Japan
}

\section{Keywords}

Right-sided ligamentum teres · Hilar cholangiocarcinoma · Simulation

\begin{abstract}
Right-sided ligamentum teres (RSLT) is a rare congenital anomaly often accompanied by variation of the hepatic vasculature. We herein report a surgical case of a hilar cholangiocarcinoma with RSLT in whom preoperative hepatectomy simulation proved useful for understanding the anatomical structure of the liver. A 78-year-old male with obstructive jaundice was referred to our department for further examination. The patient was suspected of having a hilar cholangiocarcinoma originating from the left hepatic bile duct by contrast-enhanced computed tomography (CT), and CT also showed right umbilical portion (RUP). Threedimensional images of the hepatic vasculature and biliary system reconstructed using a hepatectomy simulation system suggested that all portal branches ramified from RUP were right paramedian branches, and three leftward portal branches from these ran parallel to the peripheral bile ducts confluent with the left hepatic bile duct, where the tumor was present. Hepatic resection of part of the ventral area of the right paramedian sector and left hemiliver was performed along the demarcation line drawn after clamping the portal branches; the ratio of estimated liver resection volume was $28.9 \%$. After the operation, bile leakage oc-
\end{abstract}


Hai et al:: Hepatectomy for Hilar Cholangiocarcinoma with Right-Sided Ligamentum Teres Using a Hepatectomy Simulation System

curred. However, the leakage was treated with percutaneous drainage alone, and the patient was discharged 77 days after the operation. The patient is doing well without any signs of recurrence 21 months after the operation. The vascular and biliary anatomy in patients with RSLT is complicated and should be evaluated in detail preoperatively using a hepatectomy simulation system.

(C) 2017 The Author(s)

Published by S. Karger AG, Basel

\section{Background}

Right-sided ligamentum teres (RSLT) is a rare congenital anomaly often accompanied by variation in the hepatic vasculature $[1,2]$. Intrahepatic portal venous ramifications are more complex in such conditions, and a detailed assessment of the intrahepatic portal venous ramifications is needed when performing anatomical hepatic resection for hepatobiliary neoplasms with RSLT. In particular, successful management of hilar cholangiocarcinoma requires the accurate preoperative evaluation of the tumor spread and appropriate planning of the operative procedure; therefore, not only the hepatic vasculature but also the biliary system of the liver should be evaluated sufficiently in order to perform hepatectomy safely for cases of RSLT. A recently developed hepatectomy simulation system using multi-detector row computed tomography (CT) has enabled stereoscopic viewing of the anatomical structures of the hepatic vasculature [3]. This simulation system can also create threedimensional (3D) images of both the hepatic vasculature and the biliary system using multidetector row CT itself if the intrahepatic bile ducts are dilated.

We herein report a surgical case of a hilar cholangiocarcinoma with RSLT in whom 3D images of the hepatic vasculature and biliary system constructed using a preoperative hepatectomy simulation system were useful for understanding the anatomical structure of the liver and deciding on a surgical procedure.

\section{Case Presentation}

A 78-year-old male visited a health clinic with a chief complaint of prolonged general fatigue and brown urine for 1 month, and hyperbilirubinemia and an impaired liver function were detected on a laboratory examination. The patient was therefore referred to our department for further examination and treatment.

The patient was afebrile, but his bulbar conjunctiva was slightly yellowish. On laboratory examination, the leukocyte and C-reactive protein levels were within the normal range, but high serum concentration of total bilirubin $(3.0 \mathrm{mg} / \mathrm{dL}$; normal range, $0.2-1.2 \mathrm{mg} / \mathrm{dL}$ ), aspartate aminotransferase (126 U/L; normal range, 13-33 U/L), alanine aminotransferase (243 U/L; normal range, 8-42 U/L), alkaline phosphatase (3,070 U/L; normal range, 115$359 \mathrm{U} / \mathrm{L})$, gamma-guanosine triphosphate (3,746 U/L; normal range, 11-58 U/L), and carbohydrate antigen 19-9 (61.1 U/mL; normal range, $0-37 \mathrm{U} / \mathrm{mL})$ were revealed.

Contrast-enhanced CT demonstrated dilatation of the intrahepatic bile ducts and tumorous lesion enhanced during the arterial phase from the left hepatic bile duct to the upper extrahepatic bile duct (Fig. 1a). In addition, right umbilical portion (RUP) and left-sided gallbladder were also suggested to be present (Fig. 1b). Magnetic resonance cholangiopancreatography (MRCP) revealed stricture of the hepatic hilum to the left hepatic duct and dilatation of the intrahepatic bile duct (Fig. 1c). Endoscopic nasogastric biliary drainage was performed for obstructive jaundice and endoscopic retrograde cholangiopancreatography 
(ERCP) demonstrated no other intrahepatic bile ducts except for the bile duct in which the tip of the drainage tube had been placed (Fig. 1d). Based on these imaging findings, the patient was diagnosed with bismuth IIIb or IV type hilar cholangiocarcinoma with RSLT.

3D reconstruction of the hepatic vascular structures and hepatectomy simulation was performed using Synapse Vincent (Fujifilm Medical, Tokyo, Japan). The portal branch of the right lateral sector first diverged alone from the main portal vein, and RUP ramified into several leftward and rightward portal branches. A developed anterior fissure vein was running between these portal branches ramified from RUP, and a middle hepatic vein was seen normally (Fig. 2a). The tumor was located at the point of communication with the main hepatic duct, with significant progression to the left hepatic duct. Regarding the confluence pattern of the intrahepatic bile duct, the right hepatic bile duct was long, and the bile duct of the caudate lobe was confluent with the posterior bile duct (Fig. 2b). Based on 3D images of the hepatic vasculature and biliary system, all portal branches ramified from RUP were suggested to be right paramedian branches, and three portal branches ran parallel to the bile ducts in which the tip of the drainage tube had been placed (Fig. 2c). The perfused area of these three portal branches ramified from RUP and the left lateral and left paramedian portal branches included all peripheral bile ducts confluent with the left hepatic bile duct where the tumor was present. Therefore, extended left bisectionectomy (part of the ventral area of the right paramedian sector, left paramedian sector, and left lateral sector) without caudate lobectomy and resection of the extrahepatic bile duct was planned. The ratio of estimated liver resection volume was $28.9 \%$ (estimated liver resection volume $275 \mathrm{~mL} /$ total liver volume $951 \mathrm{~mL}$ ) according to a hepatic volumetric analysis (Fig. 2d).

On laparotomy, the gallbladder was identified to the left of the round ligament (Fig. 3a). A demarcation line corresponding to the preoperative hepatectomy simulation became apparent after the sequential clamping of the left paramedian and left lateral portal branches and the three right paramedian portal branches ramified from RUP, and hepatic parenchymal transection was performed along the demarcation line (Fig. 3b). The right hepatic duct (number of bile duct orifices in the cut end: 1 , as predicted) and common trunk of the middle and left hepatic vein were dissected, and extended left bisectionectomy with extrahepatic bile duct resection (Fig. 3c) and hepaticojejunostomy was performed as planned. Macroscopically, a hard mass was present at the hepatic hilum (Fig. 3d). Histologically, the mass lesion was diagnosed as well-differentiated tubular adenocarcinoma, and the cut end of the right hepatic duct was determined to be negative.

After the operation, bile leakage from the dissected peripheral bile duct in the ventral portion of the right paramedian sector of the liver occurred. Fortunately, the leakage was able to be treated by percutaneous drainage alone, and the patient was discharged 77 days after the operation. The patient is doing well without any signs of recurrence 21 months after the operation.

\section{Discussion}

RSLT is a rare congenital anomaly with a reported frequency of $0.2-1.2 \%[1,2,4]$, and RSLT is often accompanied by variations in the hepatic vasculature. The branching pattern in which the first portal branch runs to the right lateral sector (independent right lateral type) was reported to be more common than the bifurcated type of portal vein branching in livers with $\operatorname{RSLT}[2,4,5]$. Furthermore, intrahepatic portal venous ramifications, especially portal vein branches to Segment 4 of the liver, make the situation even more confusing. Some au- 
Hai et al.: Hepatectomy for Hilar Cholangiocarcinoma with Right-Sided Ligamentum Teres Using a Hepatectomy Simulation System

thors [6-8] have reported that Segment 4 of the liver with RSLT is supplied by branches arising from RUP. However, Shindoh et al. [4] and Gupta et al. [5] conversely reported that Segment 4 is fed by the left paramedian portal pedicle, based on their experienced cases with RSLT using 3D simulation. In addition, Shindoh et al. [4] mentioned that the basic segmental structures were as well preserved as in typical liver anatomy, although the portal vein branches to Segment 4 were suggested to be difficult to determine and the left paramedian sector was not actually subdivided into Segments 3 and 4 in the absence of the left umbilical vein $[2,5,9]$. In the present case, the ramification pattern of the main portal branch was independent right lateral type, and the leftward portal branches ramified from RUP were initially suspected to be portal branches of Segment 4 in the liver, with the intrahepatic bile duct demonstrated by ERCP believed to be a bile duct of Segment 4 based on the findings of MRCP.

In 2016, Nishitai et al. [10] identified 4 intrahepatic biliary tree confluence patterns in their analysis of cholangiograms in 46 patients with RSLT. They found that the symmetrical type, in which the right hepatic duct drains Segment $6+7$ plus the dorsal area of Segment $5+8$ and the left hepatic duct drains the ventral area of Segment $5+8$ and the left hemiliver, was the most frequent pattern. However, only a few case reports had described the biliary confluence patterns $[6,11,12]$ at the time when our patient underwent his operation, and the confluence pattern of the intrahepatic biliary system in patients with RSLT was not well understood. Therefore, the vascular and biliary structure of the present case was beyond our comprehension based solely on images from CT, MRCP, and ERCP. The use of a hepatectomy simulation system provided a better understanding of the liver anatomy and helped us plan our operative procedure.

In the present case, the 3D images constructed by hepatectomy simulation suggested that the basic liver anatomy was equivalent to the normal liver, as mentioned by Shindoh et al. [4], and all of the portal branches ramified from RUP were right paramedian branches. It was therefore reasonable to conclude that the intrahepatic bile ducts demonstrated by ERCP were right paramedian bile ducts, as these bile ducts ran parallel to some portal branches ramified from RUP, although it was impossible to identify the area and portal branches of Segment 4.

The utility of 3D imaging for evaluating the longitudinal tumor spread of hilar cholangiocarcinoma and estimating the number of bile duct orifices in the cut end of the hilar plate was recently reported $[13,14]$. Such abilities might help us plan a curative operative procedure. In the present case, hepatectomy simulation predicted the number of bile duct orifices to be 1 and suggested that the caudate lobe of the liver could be preserved, as the predicted line of transection of the right hepatic bile duct was long and the bile duct of the caudate lobe was confluent with the periphery of the right hepatic bile duct. We were therefore able to plan the optimum hepatectomy procedure using this system and performed the subsequent surgery safely by determining the transection line by clamping the portal branches according to plan, although bile leakage did unfortunately occur as a complication.

In conclusion, the vascular and biliary anatomy in patients with RSLT is complicated and should be evaluated in detail preoperatively using a hepatectomy simulation system, especially when performing a curative hepatic resection for hilar cholangiocarcinoma. 


\section{Case Reports in Gastroenterology}

Hai et al.: Hepatectomy for Hilar Cholangiocarcinoma with Right-Sided Ligamentum Teres Using a Hepatectomy Simulation System

\section{Statement of Ethics}

Written informed consent was obtained from the patient for publication of this case report and any accompanying images. A copy of the written consent is available for review by the Editor-in-Chief of this journal. The authors declare that they have no ethical conflicts.

\section{Disclosure Statement}

The authors have no conflicts of interest to disclose.

\section{References}

1 Maetani Y, Itoh K, Kojima N, Tabuchi T, Shibata T, Asonuma K, Tanaka K, Konishi J: Portal vein anomaly associated with deviation of the ligamentum teres to the right and malposition of the gallbladder. Radiology 1998;207:723-728.

-2 Nagai M, Kubota K, Kawasaki S, Takayama T, Bandai Y, Makuuchi M: Are left-sided gallbladders really located on the left side? Ann Surg 1997;225:274-280.

-3 Saito S, Yamanaka J, Miura K, Nakao N, Nagao T, Sugimoto T, Hirano T, Kuroda N, Iimuro Y, Fujimoto J: A novel 3D hepatectomy simulation based on liver circulation: application to liver resection and transplantation. Hepatology 2005;41:1297-304.

4 Shindoh J, Akahane M, Satou S, Aoki T, Beck Y, Hasegawa K, Sugawara Y, Ohtomo K, Kokudo N: Vascular architecture in anomalous right-sided ligamentum teres: three-dimensional analyses in 35 patients. HPB (Oxford) 2012;14:32-41.

5 Gupta R, Miyazaki A, Cho A, Ryu M: Portal vein branching pattern in anomalous right-sided round ligament. Abdom Imaging 2010;35:332-336.

6 Uesaka K, Yasui K, Morimoto T, Torii A, Kodera Y, Hirai T, Yamamura Y, Kato T, Kito T: Left-sided gallbladder with intrahepatic portal venous anomalies. J Hepatobiliary Pancreat Surg 1995;2:425-430.

7 Wu TC, Lee RC, Chiang JH, Chang CY: Reappraisal of left-sided gallbladder and its accompanying anomalies: a report of two cases and literature review. Acta Radiol 2005;46:233-236.

-8 Rocca JP, Rodriguez-Davalos MI, Burke-Davis M, Marvin MR, Sheiner PA, Facciuto ME: Living-donor hepatectomy in right-sided round-ligament liver: importance of mapping the anatomy to the left medial segment. J Hepatobiliary Pancreat Surg 2006;13:454-457.

-9 Ibukuro K, Takeguchi T, Fukuda H, Abe S, Tobe K: Spatial anatomy of the round ligament, gallbladder, and intrahepatic vessels in patients with right-sided round ligament of the liver. Surg Radiol Anat 2016;38:1061-1067.

10 Nishitai R, Shindoh J, Yamaoka T, Akahane M, Kokudo N, Manaka D: Biliary architecture of livers exhibiting right-sided ligamentum teres: an indication for preoperative cholangiography prior to major hepatectomy. HPB (Oxford) 2016;18:929-935.

-11 Kaneoka Y, Yamaguchi A, Isogai M, Harada T: Hepatectomy for cholangiocarcinoma complicated with right umbilical portion: anomalous configuration of the intrahepatic biliary tree. J Hepatobiliary Pancreat Surg 2000;7:321-326.

12 Hsu SL, Chen TY, Huang TL, Sun CK, Concejero AM, Tsang LL, Cheng YF: Left-sided gallbladder: its clinical significance and imaging presentations. World J Gastroenterol 2007;13:6404-6409.

13 Endo I, Shimada H, Sugita M, Fujii Y, Morioka D, Takeda K, Sugae S, Tanaka K, Togo S, Bourquain H, Peitgen HO: Role of three-dimensional imaging in operative planning for hilar cholangiocarcinoma. Surgery 2007;142:666-675.

14 Sasaki R, Kondo T, Oda T, Murata S, Wakabayashi G, Ohkohchi N: Impact of three-dimensional analysis of multidetector row computed tomography cholangioportography in operative planning for hilar cholangiocarcinoma. Am J Surg 2011;202:441-448. 
Hai et al.: Hepatectomy for Hilar Cholangiocarcinoma with Right-Sided Ligamentum Teres Using a Hepatectomy Simulation System

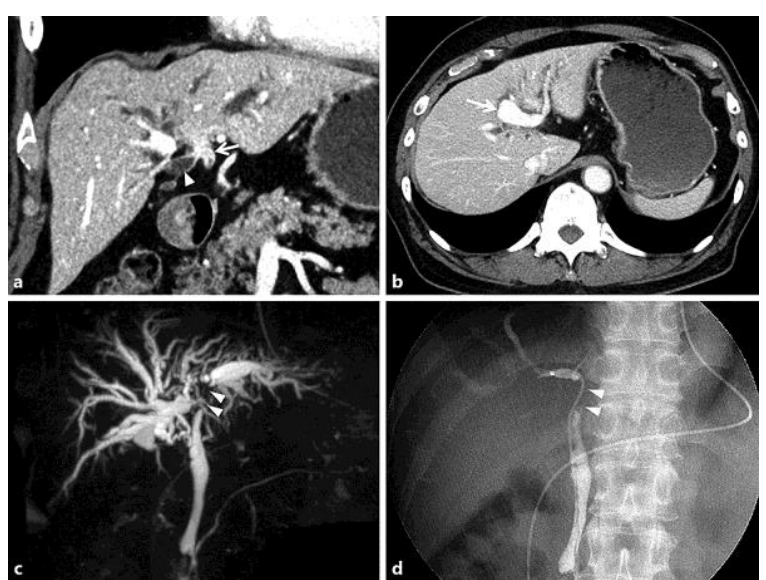

Fig. 1. Imaging series. Contrast-enhanced CT shows an enhanced tumorous lesion (arrow) at the hepatic hilum and dilated right hepatic duct (arrowhead) (a) and demonstrates right umbilical portion (b). c Bile duct from the hepatic hilum to the left (arrowheads) is not shown by magnetic resonance cholangiopancreatography. $\mathbf{d}$ Endoscopic retrograde cholangiopancreatography demonstrates stricture of the left hepatic duct (arrowheads) and a few intrahepatic bile ducts in which the tip of the drainage tube is placed. 


\section{Case Reports in Gastroenterology}
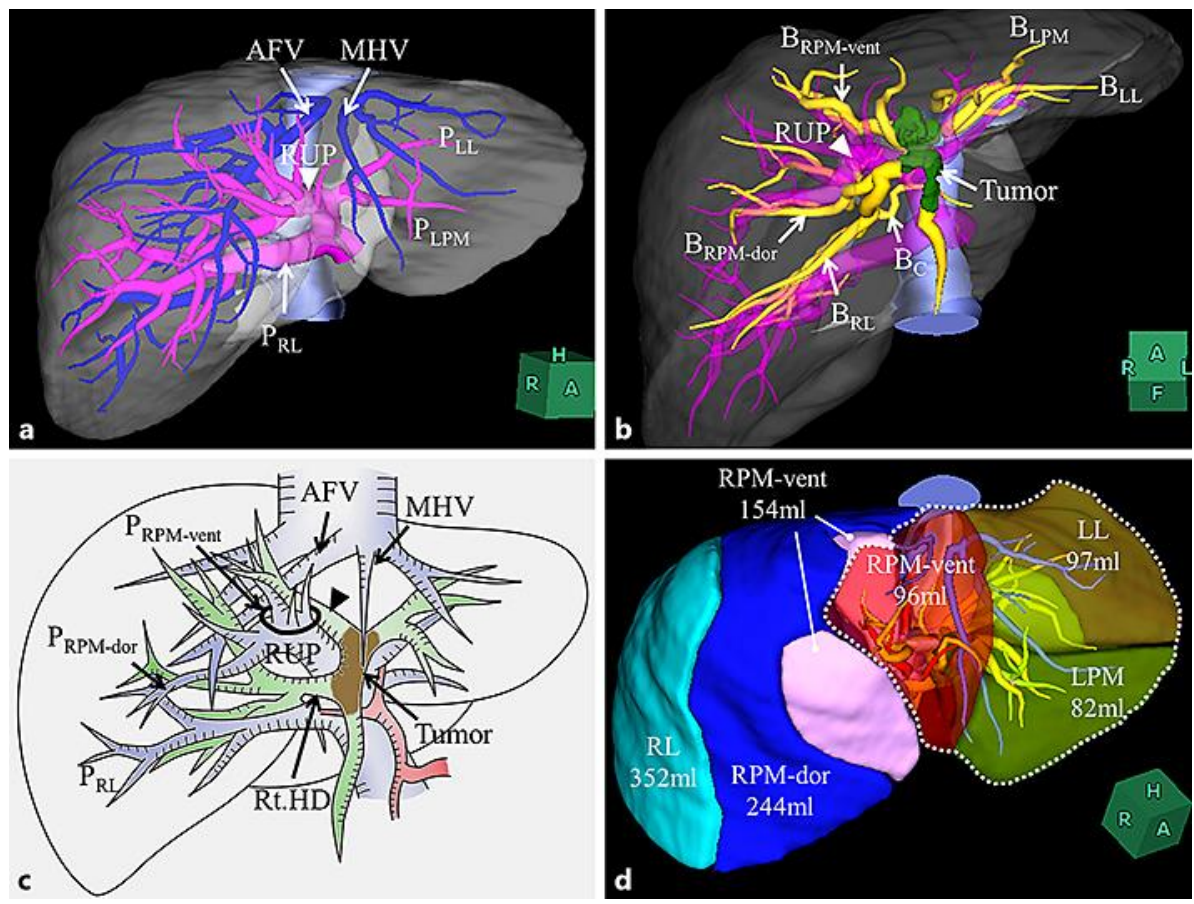

Fig. 2. a, b Images of the portal vein, hepatic vein, and biliary system reconstructed using the hepatectomy simulation system. c Schematic illustration of the liver anatomy: three ventral branches (circle) of the right paramedian portal veins ramified from RUP run parallel to the intrahepatic bile duct (arrowhead) demonstrated by endoscopic retrograde cholangiopancreatography. $\mathbf{d}$ Hepatectomy simulation: the perfused area (white dotted line) included all peripheral bile ducts confluent with the left hepatic bile duct, where the

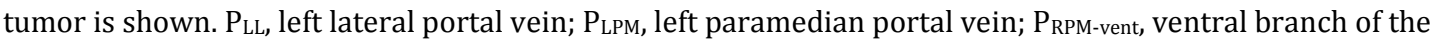
right paramedian portal vein; $\mathrm{P}_{\mathrm{RPM}-\mathrm{dor}}$, dorsal branch of the right paramedian portal vein; $\mathrm{P}_{\mathrm{RL}}$, right lateral portal vein; RUP, right umbilical portion; AFV, anterior fissure vein; MHV, middle hepatic vein; $\mathrm{B}_{\mathrm{LL}}$, left

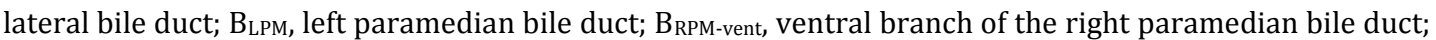

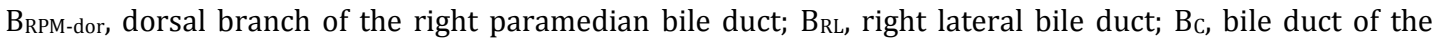
caudate lobe; Rt.HD, right hepatic duct; LL, left lateral sector; LPM, left paramedian sector; RPM-vent, ventral area of the right paramedian sector; RPM-dor, dorsal area of the right paramedian sector; RL, right lateral sector. 


\section{Case Reports in Gastroenterology} Case Rep Gastroenterol 2017;11:576-583 DOI: $10.1159 / 000480375$

(C) 2017 The Author(s). Published by S. Karger AG, Basel www.karger.com/crg

Hai et al.: Hepatectomy for Hilar Cholangiocarcinoma with Right-Sided Ligamentum Teres Using a Hepatectomy Simulation System
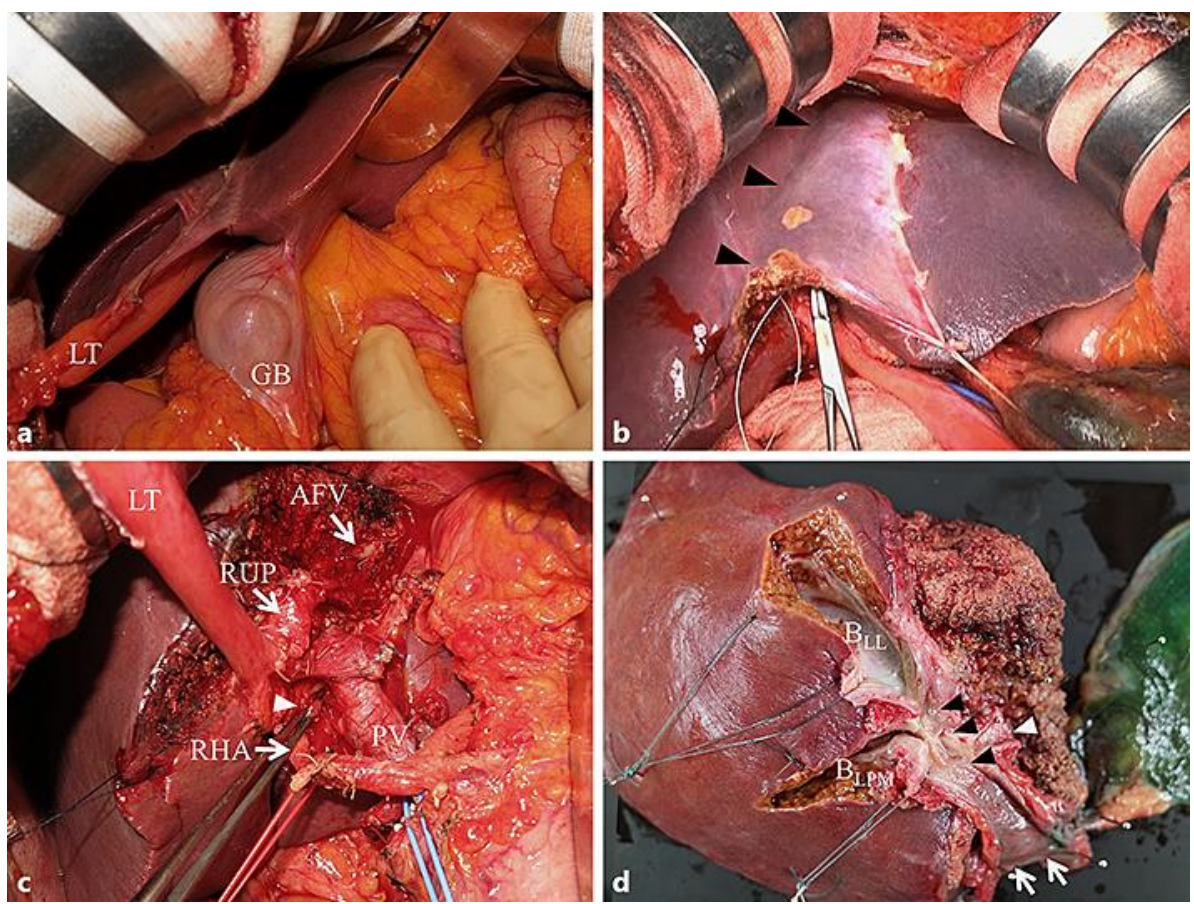

Fig. 3. Intraoperative findings. The round ligament is positioned at the right side of the gallbladder (GB) (a). The demarcation line (arrowheads) is clear after dissection of the left paramedian portal branch, left lateral portal branch, and three right paramedian portal branches (b). $\mathbf{c}$ The operative field after extended left bisectionectomy with extrahepatic bile duct resection (arrowhead: stump of right hepatic duct). d Macroscopic examination shows an elevated lesion (black arrowheads) at the hepatic hilum (white arrowhead: stump of right hepatic duct, arrows: stump of common bile duct). LT, ligamentum teres; RUP, right umbilical portion; AFV, anterior fissure vein; RHA, right hepatic artery; PV, portal vein; BLL, left lateral bile duct; BLPM, left paramedian bile duct. 